\title{
Radioisotope Production through Accelerators in Crystalline Targets ${ }^{\dagger}$
}

\author{
Giacomo Germogli 1,*, Davide De Salvador 2,3, Luca Bacci ${ }^{3}$, Enrico Bagli ${ }^{1}$, Laura Bandiera ${ }^{1}$, \\ Riccardo Camattari ${ }^{1}$, Sara Maria Carturan ${ }^{3}$, Davide Casotti ${ }^{1}$, Federico Evangelisti ${ }^{1}$, \\ Giacomo Guidi 4, Vincenzo Guidi 5, Gianluigi Maggioni ${ }^{3}$, Tais Maiolino ${ }^{1}$, Andrea Mazzolari ${ }^{1}$, \\ Roberto Menegazzo 6, Daniel Ricardo Napoli ${ }^{2}$, Walter Raniero ${ }^{2}$, Marco Romagnoni 5, \\ Carlo Scian ${ }^{3}$, Alexei Sytov ${ }^{1}$, Jose' Javier Valiente Dobón ${ }^{2}$ and Donato Vincenzi ${ }^{5}$ \\ 1 INFN Section of Ferrara, via Saragat 1, 44122 Ferrara, Italy \\ 2 INFN Legnaro National Laboratories, Viale dell'Università 2, 35020 Legnaro (PD), Italy \\ 3 Dipartimento di Fisica e Astronomia “Galileo Galilei”, via Marzolo 8, 35131 Padova, Italy \\ 4 Comecer S.p.A., Via Maestri del Lavoro, 90, 48014 Castel Bolognese (RA), Italy \\ 5 Dipartimento di Fisica e Scienze della Terra, Via Saragat 1, 44122 Ferrara, Italy \\ 6 INFN Section of Padova, via Marzolo 8, 35131 Padova, Italy \\ * Correspondence: germogli@fe.infn.it \\ + Presented at the 37th International Symposium on Dynamical Properties of Solids (DyProSo 2019), \\ Ferrara, Italy, 8-12 September 2019. \\ Published: 5 September 2019
}

\begin{abstract}
The production techniques of radioisotopes for medical purposes is a valuable and important field in nuclear medicine. In particular, the expensive cost of the prime materials for the production via cyclotron obliges the search for new solutions to enhance the production rate with minor upgrades of the current instrumentation. Oriented ordered structure can modify particle trajectories inside a medium leading to a sensible variation of the interaction rate with atomic nuclei. Under specific orientations of the target with respect to the incident beam, the probability of inelastic interaction with nuclei can be enhanced with respect to the standard rate. This effect is called antichanneling and leads to an increase of the radioisotope production yield. A dedicated set of experimental measurements were carried out at the $\mathrm{CN}$ accelerator of the INFN Legnaro Laboratories to investigate nuclear reactions under channeling experiments. In particular, the production of the Arsenic-74 radioisotope through a proton beam delivered to a natural singlecrystal Germanium target was monitored via $\gamma$-spectroscopy of the prompt $\gamma$-rays upon deexcitation of produced nuclei, in order to quantify the production rate variation as a function of the incident angle.
\end{abstract}

\section{Introduction}

The current wealth of radioisotopes has been made possible especially through nuclear reactors. This allowed the development of several applications in modern nuclear medicine, biology, and other fields. At the same time, great efforts have been made for the development of accelerators devoted to the production of radioisotopes [1]. Indeed, production through accelerators is spreading worldwide, despite its higher cost with respect to the production via nuclear reactors, due to some advantages such as the high specific activity that can be obtained through the $(p, x n)$ and $(p, \alpha)$ reactions, the smaller amount of radioactive waste, and the possibility to produce radioisotopes very close to hospitals in compact facilities.

In this contest, the research of new technological solutions to obtain a more efficient radioisotope production is of special interest. One possible way to improve production efficiency with a minor upgrade of the existing technology is the usage of a crystalline target. Indeed, ordered and oriented 
structures, such as crystals, can modify the trajectories of incoming beam particles interacting with them. Compared to the motion into an amorphous medium, ions interacting with a crystal are subject to coherent interactions due to the periodicity of the atoms in the crystalline structure. This phenomenon is capable of modifying particle trajectories inside the medium, causing the variation of the interaction rate with the atomic nuclei.

An interesting effect can be observed when charged particles enter a crystal at an angle close to the critical angle for channeling $\theta$ c with respect to an atomic plane [2] ( $\theta_{C}=\sqrt{U / E}$, which depends on the interplanar potential well with depth $U$, and the particle energy $E$ ). In such a case, almost all the beam particles, even those entering in the middle of two planes, undergo a motion above the maxima of the potential barriers, where the nuclei of the lattice atoms are located, thus resulting in a higher probability of interaction with nuclei with respect to the motion into an amorphous medium with the same density [3]. In particular, the enhancement of the ${ }^{18} \mathrm{O}(\mathrm{p}, \alpha)^{15} \mathrm{~N}$ reaction obtained with protons at $642.5 \mathrm{keV}$ (i.e., just above a sharp resonance at $628 \mathrm{keV}$ ) impinging a $\mathrm{Al}_{2} \mathrm{O}_{3}$ crystal has been observed through a dedicated experiment performed at the INFN-Legnaro Laboratories [4]. This result suggested the development of a crystalline target for the production of radioisotopes of interest for medical applications. In this paper, a measurement of the enhancement of the production of the Arsenic-74 isotope, which is used in tumor imaging [5], via the ${ }^{74} \mathrm{Ge}(\mathrm{p}, \mathrm{n})^{74} \mathrm{As}$ reaction in antichanneling condition, is shown.

\section{The Experimental Setup}

The LNL-CN is a Van Der Graaf electrostatic accelerator with a maximum voltage terminal of 5.5 MV, thus being ideal for investigating nuclear reactions in the first layers of a material. As shown in Figure 1, a rectangular Ge sample $(10 \times 10 \times 1 \mathrm{~mm}$, with the main surface oriented along the (111) crystallographic planes) is installed onto a goniometer with angular resolution of $0.01^{\circ}$ and a minimum pressure inside the chamber of approximately $10^{-7} \mathrm{mbar}$. This goniometer allowed the alignment of the crystal axis with respect to the incoming beam, to study the transition from channeling to antichanneling condition. Beam divergence and goniometer resolution-both of the order of $0.01^{\circ}$-are perfectly suitable for channeling studies at these energies, taking into account that $\theta c$ for channeling between $\mathrm{Ge}(100)$ planes for $4 \mathrm{MeV}$ protons is of the order of $0.2^{\circ}$.

The alignment of the samples was done through a silicon detector for Rutherford Backscattering analysis (RBS), i.e., by measuring the backscattered particles as a function of the angle between the beam and the samples. The RBS detector was placed at $115 \mathrm{~mm}$ and at an angle of $160^{\circ}$ from the target. A HPGe detector was placed outside the chamber in order to measure the prompt $\gamma$-radiation emitted by the target during the irradiation with the proton beam.

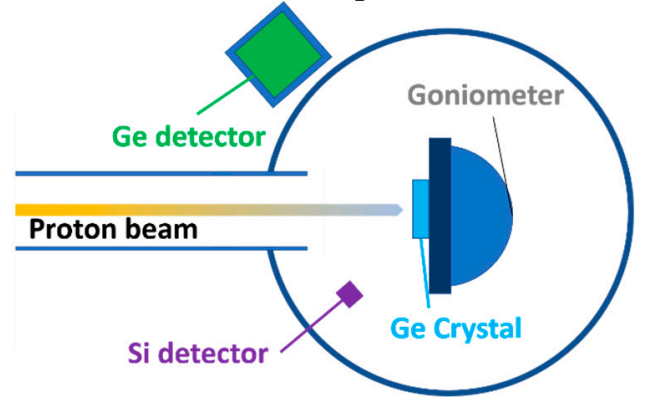

Figure 1. Experimental setup. A proton beam is delivered by the LNL-CN accelerator to a germanium single crystal installed onto a goniometer. Backscattered protons are detected through a silicon detector, while the $\gamma$-radiation is measured through a HPGe detector.

The beam energy was set at $3.80 \mathrm{MeV}$, i.e., a value slightly above the threshold of production of the Arsenic-74 radioisotope through the ${ }^{74} \mathrm{Ge}(\mathrm{p}, \mathrm{n})^{74} \mathrm{As}$ reaction, in order to confine the production of the radioisotope in the first few micrometers of thickness of the target, where the antichanneling effect is concentrated. The amount of ${ }^{74} \mathrm{As}$ produced can be determined by considering the prompt $\gamma$-rays 
from the low-lying excited states of the ${ }^{74} \mathrm{As}$ in the measured $\gamma$-spectra. The scheme of the nuclear reaction and of the $\gamma$-radiation studied is shown in Figure. 2

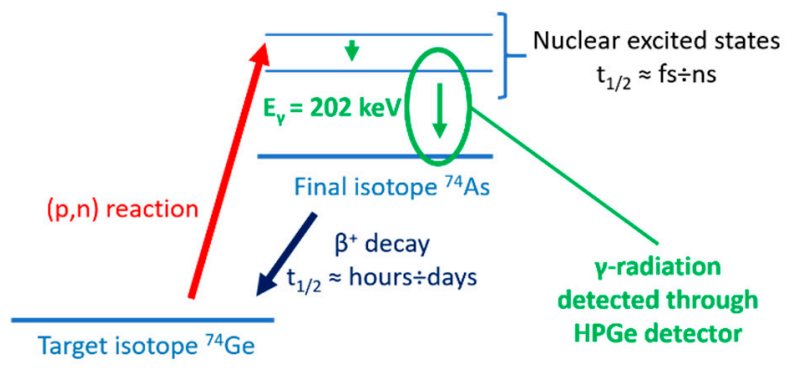

Figure 2. Scheme of the nuclear reaction studied. Prompt $\gamma$-radiation from the low-lying $202 \mathrm{keV}$ state of ${ }^{74} \mathrm{As}$ is measured through the HPGe detector to determine online the ${ }^{74} \mathrm{As}$ production rate.

\section{Results}

Firstly, the threshold energy for protons for populating the low-lying excited levels of Arsenic74 was determined. For this purpose, measurements of the $\gamma$-spectra for a proton beam at different energies $(3.75,3.80$ and $3.85 \mathrm{MeV})$ were performed. In particular, by looking at the integral of the $\gamma-$ peak at $202 \mathrm{keV}$ (see Fig. 3b, as an example, the $\gamma$-spectrum for protons at $3.80 \mathrm{MeV}$ ) emitted by the excited ${ }^{74} \mathrm{As}$ we extrapolated a threshold energy $E_{T h}=3.72 \pm 0.01 \mathrm{MeV}$. This result allowed to find the maximum depth for the production reaction through protons incident at $E_{I n}=3.80 \mathrm{MeV}$ by means of a simulation of the energy loss of protons into germanium via SRIM software, obtaining a depth $d_{\mathrm{Th}}$, $E i n=3.80 \mathrm{keV}=2.75 \pm 0.05 \mu \mathrm{m}$. Then, those protons which suffer backscattering into the target as they are at the threshold energy, will reach the Si detector having an energy of $E_{T h, R B S}=3.44 \pm 0.01 \mathrm{MeV}$. As shown in Fig. 3(a), after finding the alignment for axial channeling condition in which backscattering is at its minimum (black line), a comparison of the integral of the RBS spectra above the $E_{T h, R B S}$ energy shows an enhancement in backscattering efficiency in antichanneling condition of $9.1 \pm 0.3 \%$ with respect to the randomly oriented case. At the same time, the integral of the $202-\mathrm{keV}$ peak (see Figure 3(b)) shows that the production of the ${ }^{74} \mathrm{As}$ in antichanneling is increased of the $9.8 \pm 0.6 \%$ with respect to the random case.
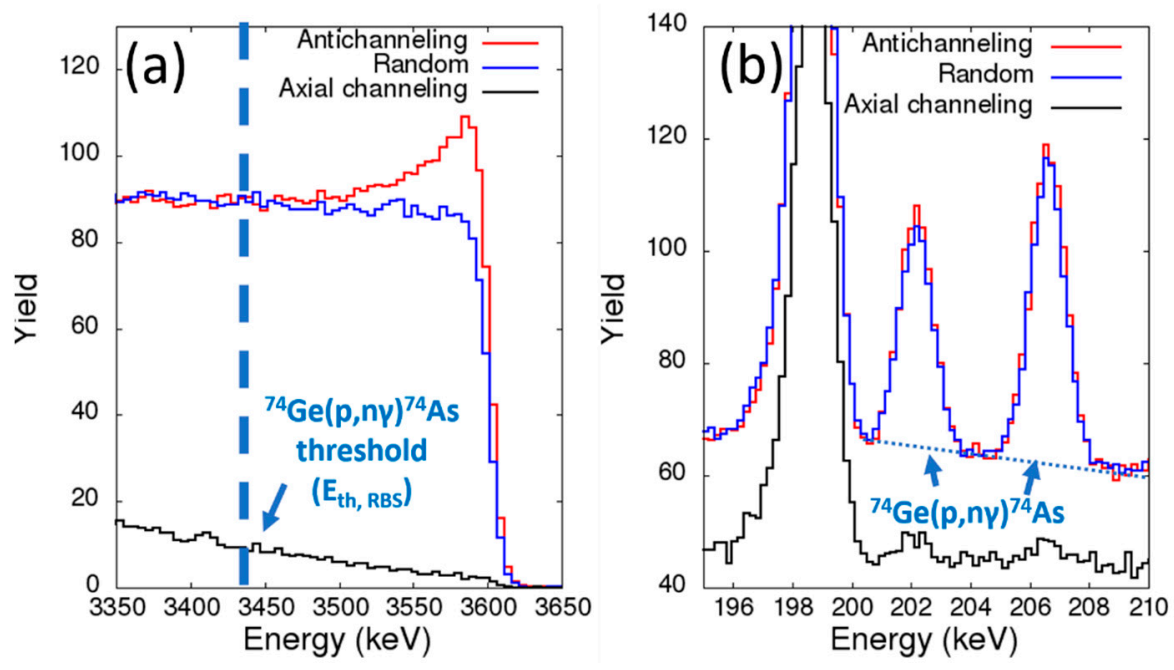

Figure 3. Rutherford Backscattering of protons incident at $3.80 \mathrm{MeV}$ (RBS, a) and $\gamma$-radiation (b) measured online at different alignment conditions (antichanneling, random orientation, axial channeling). Yield is normalized to $1 \mu \mathrm{C}$ of incoming protons. 


\section{Conclusions}

In this work, the enhancement of the production of a radioisotope for medical applications due to the antichanneling effect is shown. The measurement was performed through $\gamma$-spectroscopy online, i.e., during the irradiation of the target with a proton beam, after performing the alignment of the target by means of the Rutherford Backscattering of the proton beam. In particular, the increase of the amount of backscattered protons above the reaction threshold in antichanneling condition with respect to the case of a randomly oriented target is found to be in good agreement with the enhancement of the production of the Arsenic-74 radioisotope, as one can observe looking at the 202 $\mathrm{keV}$ line in the $\gamma$-spectra. Both the effects are due to the higher nuclear density experienced by the beam particles undergoing the quasi-channeled motion. This result confirms the possibility to improve the production of radioisotopes for medical applications via antichanneling.

Funding: We acknowledge the financial support of the European Regional Development Fund through the PORFESR 2014-2020 programme (INFN-TTLAB TROPIC project, CUP I32F17000440002) and of the INFN CSNVGECO project.

\section{References}

1. Schmor, P., Review of Cyclotrons for the Production of Radioactive Isotopes for Medical and Industrial Applications. Rev. Accelerator Sci. Technol. 2012, 4, 103-116, doi:10.1142/9789814383998_0005.

2. Lindhard, J. Influence of crystal lattice on motion of energetic charged particles. Kongel. Dan. Vidensk. Selsk. Mat.-Fys. Medd. 1965, 34. Available online: http://gymarkiv.sdu.dk/MFM/kdvs/mfm\%2030-39/mfm-3414.pdf (accessed on 1 Augest 2019).

3. Smulders, P.J.M.et al., Giant focusing peak and potential dependence observed in a transition from axial to planar channeling in Si., Nucl. Instr. and Meth. in Phys. Res. B 1992, 67, 185-188.

4. Bagli, E.; de Salvador, D.; Bacci, L.; Sgarbossa, F.; Bandiera, L.; Camattari, R.; Germogli, G.; Mazzolari, A.; Sytov, A.; Guidi, G.; Enhancement of the Inelastic Nuclear Interaction Rate in Crystals via Antichanneling, Phys. Rev. Lett. 2019, 123, 044801.

5. Jennewein, M.; Lewis, M.A., Zhao, D.; Tsyganov, E.; Slavine, N.; He, J.; Watkins, L.; Kodibagkar, V.D.; O'Kelly, S.; Kulkarni,P.; et al., Vascular Imaging of Solid Tumors in Rats with a Radioactive ArsenicLabeled Antibody that Binds Exposed Phosphatidylserine. Clin. Cancer Res. 2008, 14, 1377-1385.

(C) 2019 by the authors. Licensee MDPI, Basel, Switzerland. This article is an open access article distributed under the terms and conditions of the Creative Commons Attribution (CC BY) license (http://creativecommons.org/licenses/by/4.0/). 